\title{
Deceptive Redistribution*
}

\author{
Simeon D. Alder \\ University of Notre Dame
}

\author{
Guillermo L. Ordoñez \\ Yale University
}

February 2011

\section{PRELIMINARY AND INCOMPLETE - PleAse Do Not Cite}

\begin{abstract}
While economic and redistributive policies can be welfare enhancing in an environment characterized by market failures and inequality, they frequently generate private gains to those who hold public office. In a setting with dispersed information about the policies' true motives we ask how self-interested governments who fret over their perceived integrity (reputation) balance legitimate needs for government action with the temptations of rent-seeking. Compared to the previous literature our model generates a richer trade-off structure between redistribution and efficiency. We find that governments use transfers strategically to conceal inefficient policy choices and excessive office rents. Our model also offers novel economic insights into the role of information frictions in shaping the governments' political accountability.
\end{abstract}

*An earlier version of the paper circulated under the title "Endogenous Coalitions". For helpful comments we would like to thank seminar participants at Notre Dame, the Federal Reserve Bank of St.Louis, and Western Ontario. 


\section{Introduction}

Across time and space one can find countless examples of socially inefficient policies. What's more, one can convincingly argue that their longevity is fueled - at least in part - by private benefits accruing to those with policy-making authority. Often, individuals or groups of agents do not have information that is precise enough to ascertain whether a particular policy is indeed warranted by an alleged social concern - such as the need to correct a market failure, for instance - or by more narrow interests. Since many policies target specific activities or agents (sectors, industries, age groups, or likely voters, to mention just a few) more precise knowledge alone may not be enough to prevent governments from behaving opportunistically, whenever the target's political clout is limited.

From the observation that public policies are always "marketed" in terms of their social benefits rather than the private gains we conclude that governments care about their reputation in the sense that they do not want to be seen as excessively opportunistic for fear of being removed from office.

Our work offers valuable insights into the incentives and constraints of governments who are not held accountable politically in quite the same way as those in mature democracies. In particular, our model characterizes the conditions under which the disciplining power of the government's reputation concerns is strengthened or weakened. In addition, our work highlights what governments can do to "manage" their reputation and thereby mitigate the political and economic consequences of opportunistic behavior.

We develop a reputational model of government with information frictions. The government's actions are revealed asymmetrically to (groups of) citizens who are economically productive voters. Governments have an interest in selecting inefficient policies in their attempt to maximize rents associated with holding office. However, since well-informed groups can share information with less knowledgeable ones by way of costly signals, the prospect of facing a coalition of dissatisfied rioters muffles the government's incentive to extract excessive office rents.

In this framework we show that redistribution can be used to undermine the formation of coalitions. Given that different sectors are subject to asymmetric shocks which are independent of the process governing the externality, they generate an orthogo- 
nal motive for redistribution. Since the realizations are observed privately, however, the government's transfer motives become more obscure. In fact, the government can abuse such transfers to conceal rent extraction efforts in the name of redistribution. This essentially amounts to a subtle "silence-for-money" deal to choke off potential riots.

This setup enables us to replicate the stylized fact that a particular public policy may be justified on more than one ground. In Switzerland, for example, direct payments to farmers are justified as a means to alleviate rural poverty while output or sizedependent subsidies allegedly compensate them for their contribution to the conservation of the countryside (Landschaftspflege). Alternatively, given the farmers' disproportionate political influence, the direct payments may also be motivated by the need to garner their support in elections and referenda. ${ }^{1}$ Importantly, since transfers are distributed lump-sum, there are no efficiency concerns in the redistribution dimension of the model. That is not to say that we do not care about the underlying motives for transfer payments. Quite to the contrary, the extent to which citizen voters can disentangle the two rationales determines - among others - how quickly they update their beliefs about the quality of the incumbent government.

In a nutshell, the model captures the observation that few governments are unequivocally incompetent or predatory. They do, often enough it seems, respond to "legitimate" calls for government action, which insulates them somewhat from being sanctioned too harshly for inefficient policy choices. ${ }^{2}$ Our aim is to highlight how information frictions combined with costly political actions can enable governments to misbehave with relative impunity.

Unlike Besley and Coate $(1997,1998)$ and Caselli and Morelli (2004) we do not model the process by which citizens declare themselves candidates and how a particular (head of) government is elected. Rather, we assume that citizens cannot choose the type of incoming government. Their perceptions of the government's integrity or quality, however, evolve over time based on observables and noisy signals. Rather than modeling the entry margin, we focus on the exit mechanism. Depending on

\footnotetext{
${ }^{1}$ For a similar discussion about the US farm lobby the interested reader may want to refer to Acemoglu and Robinson (2001).

${ }^{2}$ There are, of course, examples of "degenerate" governments in places such as North Korea or the Belgian King Leopold II's Congo Free State. Our model, however, has nothing to say about such extreme outliers. Acemoglu (2006) discusses inefficient institutions and policies in the context of a holdup problem.
} 
the belief about the government's type citizens may resort to civil unrest (which we label as "riots") in order to transfer policy-making power from the incumbent to a new government (of uncertain type). While Caselli and Morelli (2004) find that lowquality citizens have a comparative advantage in elective office, our model suggests (in an extension yet to be written) that governments have incentives to be "populist" whenever unproductive agents have a comparative advantage in "producing" votes rather than final goods. ${ }^{3}$

This model also makes a novel contribution to the coalition literature. While Aumann and Myerson (1988); Ray and Vohra (1997, 1999); Carraro (2003); and Ray (2007) are concerned with intra-coalitional bargaining equilibria, we focus on the non-cooperative endogenous formation of new coalitions. ${ }^{4}$ Members self-select into a coalition if their beliefs about the government type are sufficiently synchronized, subject to the constraints imposed by costly action/membership.

To the extent that we populate a political economy with a government that is concerned with reputation our work is related to Coate and Morris (1995). Their work is concerned with efficient vs. inefficient forms of transferring resources between agents. Our focus, on the other hand, is on the extent to which (efficient) transfer payments mask other inefficient policies and hence affect how "precisely" citizens can update their beliefs about the government type (quality).

There is, of course, a vast literature on the age-old trade-off between redistribution and efficiency (see, for instance, Alesina and Rodrik, 1994; Persson and Tabellini, 1994, among many others). While the early literature argues that inefficiency is a necessary condition for redistribution, we find that the latter masks - and thereby enables the government to "get away" with - inefficient policies.

The remainder of the paper is organized as follows. Section 2.1 sets up the environment. In particular, we specify the informational frictions and the timing of events. Section 2.2 describes how different agents Bayesian update their beliefs about the quality of the government. In section 2.3 we define and characterize the equilibrium. Moreover, we discuss some interesting comparative statics. In section 3 we introduce an asymmetric endowment shock. Section 4 concludes and discusses future work, including the empirics to discipline the parametrization of the model.

\footnotetext{
${ }^{3}$ These populist tendencies will be dampened endogenously by other features of the model.

${ }^{4}$ Since the coalition members' resources are not pooled we can abstract from intra-coalitional bargaining altogether.
} 


\section{Effect of Asymmetric Information on Efficiency}

\subsection{Environment}

In the following sections, we introduce the model's three elementary ingredients, namely (1) the population, preferences, technology, and the stochastic events, (2) the information structure, and (3) the timing assumptions.

\subsubsection{Population, Technology, and Nature}

At any given time, the model economy is populated by a government and by $I$ cohorts indexed by $i$, each with a measure of agents $L_{i}$. We assume, for the time being, that $I=2, i \in\{Y, O\}$ indexing the young and old generations, respectively, and $L_{O}=L_{Y}$. All agents have linear preferences over consumption and are hence riskneutral.

Without loss of generality we assume that $O$ has access to a technology to produce a single consumption good using $L_{O}$ units of labor input. ${ }^{5} Y$, on the other hand, produces no output at all. ${ }^{6}$ Production by $O$ is subject to a market failure $\beta \in\left\{\beta_{L}, \beta_{H}\right\}$. For simplicity of exposition, we focus on a production externality. ${ }^{7}$ Each period, $\beta_{L}$ is realized with probability $\gamma$. In the absence of government intervention, we assume that $\beta$ leads to inefficiencies in the allocation of productive resources. Importantly, the externality is confined to production alone.

The government can be one of two types: $G$ (for good, or benevolent) or $B$ (for bad, or opportunistic). Government "quality" is drawn from an exogenous distribution with $\operatorname{Pr}(G)=\phi_{0}$.

Type- $G$ governments are concerned with social welfare. They can always implement a Pigouvian $\operatorname{tax} \alpha \in\left\{\alpha_{L}, \alpha_{H}\right\}$ to restore Pareto-optimal allocations in production. Aggregate output is a function of both the externality $\beta$ and the tax $\alpha$ and can be

\footnotetext{
${ }^{5}$ Labor supply is inelastic.

${ }^{6}$ We could, of course, assume that $Y$ has access to an alternative and less advanced technology. Qualitatively, our results would be unaffected.

${ }^{7} \mathrm{~A}$ discussion of other market failures - such as a (natural) monopoly or crony capitalism - is available upon request.
} 
ordered as follows:

$$
X_{\beta_{L}, \alpha_{L}}>X_{\beta_{L}, \alpha_{H}}>X_{\beta_{H}, \alpha_{H}}>X_{\beta_{H}, \alpha_{L}}
$$

In addition, $G$ types assign equal Pareto weights to both cohorts and transfer $T_{Y \mid \alpha_{i}}=$ $\frac{X_{\beta_{i}, \alpha_{i}}}{2}$ with $i \in\{L, H\}$ to the young lump-sum in order to equalize marginal utilities between the two generations.

Type- $B$ governments, on the other hand, trade off short-term rent extraction with a longer-term concern for their reputation when choosing a policy response to the stochastic externality. Importantly, the tax that restores first-best allocations is always in the set of available policies and $B$ types could, if they so desired, mimic a $G$ type. We characterize the conditions under which reputational concerns prevent $B$ types from behaving opportunistically.

\subsubsection{Information Structure}

The realization of the externality is observed by the old $(O)$ and by the government. The young cohort $(Y)$, on the other hand, does not observe $\beta$. This is the key information friction in the model and generates the sort of coordination failures we are interested in.

The government's type is private information. Cohort $O$ updates its belief by observing the realization of the shock $\beta$ and the government's policy $\alpha . O$ can issue a signal about the government type to $Y$ by way of a costly riot. In addition to all public pieces of information, $Y$ takes $O$ 's decision into account in the update of its belief. Its decision to riot, moreover, depends on the cohort-specific $\operatorname{cost} C_{Y}$. The $\operatorname{costs} C_{Y}$ and $C_{O}$ are drawn independently from a know distribution $F$.

Importantly, we assume that a riot by $O$ alone is not sufficient to "impeach" the incumbent government. Throwing out the government requires a majority of cohorts, i.e. both of them when $I=2 .^{8}$

In addition to $\alpha$, the lump-sum resource allocations $T_{O \mid \alpha}$ and $T_{Y \mid \alpha}$ are public information. Since $\beta$ is observed privately, the young cannot infer the true $X_{\beta, \alpha}$ from $\alpha$ and $T_{\cdot \mid \alpha}$ alone.

\footnotetext{
${ }^{8}$ With $I=2$, we need not worry about simple vs. qualified majority since in either case both sectors are required to participate in order to unseat the government.
} 


\subsubsection{Timing}

At the beginning of each period, the economy consists of a government - with reputation $\phi$-and two sectors: $O$ is the previous period's $Y$ cohort; the current $Y$ is a newborn cohort. ${ }^{9}$ The timing of subsequent shocks and actions is as follows:

1. Nature moves:

(a) Draws action costs (i.e. the cost of rioting) for each cohort from a known exogenous distribution with cumulative distribution function $F$. Each cohort's cost draw is independent, observed privately, and denoted by $C_{O}$ and $C_{Y}$, respectively.

(b) Nature also draws the externality shocks $\beta$ for cohort $O$.

2. The government observes $\beta$ and chooses a policy $\alpha$. We denote the government's strategy by

$$
\begin{aligned}
& \tau_{X}=\operatorname{Pr}\left(\alpha_{H} \mid \beta\right), \text { where } \beta=\left(\begin{array}{c}
\beta_{L} \\
\beta_{H}
\end{array}\right) \\
& \tau_{X}: \quad \beta \rightarrow[0,1]
\end{aligned}
$$

It also announces lump sum transfers to allocate resources to the young (denoted by $\left(T_{Y \mid \alpha}\right)$ and to the old $\left(T_{O \mid \alpha}\right)$. Importantly, $G$ types propose a unique bundles of $\alpha$ and a $\left(T_{Y \mid \alpha}, T_{O \mid \alpha}\right)$-pair. This feature allows us to condition on $\alpha$ alone - rather than the triple $\left(\alpha, T_{Y \mid \alpha}, T_{O \mid \alpha}\right)$ - when necessary.

3. Cohort $O$ observes $\beta, \alpha$ (as well as $\left.T_{Y \mid \alpha}, T_{O \mid \alpha}\right)$. It decides whether to $\operatorname{riot}\left(R_{O}=1\right)$ or not $\left(R_{O}=0\right)$, taking into account all relevant information, including $C_{O}$. Again, we use the following notation to describe the decisions:

$$
\begin{aligned}
& \tau_{O}=\operatorname{Pr}\left(R_{O}=1 \mid \beta, \alpha\right) \\
& \tau_{O}: \quad \beta \times \alpha \rightarrow[0,1]
\end{aligned}
$$

4. Cohort $Y$ observes $R_{O}, \alpha$ (as well as $T_{Y \mid \alpha}, T_{O \mid \alpha}$ ) and chooses the probability of rioting $\left(R_{Y}=0\right)$ or $\operatorname{not}\left(R_{Y}=1\right)$ :

\footnotetext{
${ }^{9}$ At time 0 , the reputation is always given by $\phi=\phi_{0}$.
} 


$$
\begin{aligned}
& \tau_{Y}=\operatorname{Pr}\left(R_{Y}=1 \mid R_{O}, \alpha\right) \\
& \tau_{Y}: \quad \alpha \times\{0,1\} \rightarrow[0,1]
\end{aligned}
$$

5. (a) If $R_{O}=1$ and $R_{Y}=1$, the current government pays a penalty $P$ and is replaced with a new government, which is of type $G$ with the exogenous probability $\phi_{0}$. The cost of action defaults to $C_{O}=+\infty$ (to rule out further riots in the remainder of the period). The new government chooses policy $\hat{\alpha}$ and production takes place under that policy.

(b) If $R_{O}=0$ and/or $R_{Y}=0$, production takes place under the policy $\alpha$. Moreover, the incumbent government chooses its own consumption and distributes $T_{O \mid \alpha}$ and $T_{Y \mid \alpha}$.

\section{Cohort $O$ disappears. ${ }^{10}$}

7. Youngsters $(Y)$ age and turn old $(O)$ at the end of the period. Next period's prior about the quality of the government is given by $\phi=\phi_{Y}^{\prime}$ (i.e. the posterior of today's young). A new cohort of youngsters is born; they "inherit" the prior $\phi=\phi_{Y}^{\prime}$ from their predecessors.

\subsection{Beliefs}

The decision of each cohort to riot in order to unseat the government depends on their respective beliefs about government quality. Cohort $Y$ observes the policy $\alpha$ and $R_{O}$ (the old's decision to riot or not) and updates its belief $\operatorname{Pr}\left(G \mid R_{O}, \alpha\right)$ about the government type, in order to choose $R_{Y} \in\{0,1\}$. Cohort $O$, in turn, anticipates $Y^{\prime}$ s update and forms expectations about $R_{Y}$ in its own riot decision. The government, finally, forms expectations about the actions of both generations in its policy choice.

Since we assume that $\beta \in\left\{\beta_{L}, \beta_{H}\right\}$, we can limit ourselves to four updates, conditional on $\left\{R_{O}=1, \alpha_{H}\right\},\left\{R_{O}=1, \alpha_{L}\right\},\left\{R_{O}=0, \alpha_{H}\right\}$, and $\left\{R_{O}=0, \alpha_{L}\right\}$, where $\alpha_{H}$ is the policy that implements first-best allocations when $\beta=\beta_{H}$ and $\alpha_{L}$ does the same

\footnotetext{
${ }^{10}$ This assumption allows us to eliminate higher order beliefs.
} 
under $\beta_{L} \cdot{ }^{11}$ Before we characterize the equilibrium by backward induction, we make explicit the Bayesian updating in all four cases. The details about how to obtain them are in the Appendix.

$$
\begin{aligned}
& \operatorname{Pr}\left(G \mid R_{O}=0, \alpha_{H}\right)=\frac{\phi_{\alpha_{H}}}{\phi_{\alpha_{H}}+\left[1-\frac{(1-\gamma) \tau_{O} \tau_{X}}{(1-\gamma) \tau_{X}+\gamma}\right]\left(1-\phi_{\alpha_{H}}\right)} \\
& \operatorname{Pr}\left(G \mid R_{O}=0, \alpha_{L}\right)=\frac{\phi_{\alpha_{L}}}{\phi_{\alpha_{L}}+\left(1-\phi_{\alpha_{L}}\right)}=\phi_{\alpha_{L}} \\
& \operatorname{Pr}\left(G \mid R_{O}=1, \alpha_{L}\right)=0 \\
& \operatorname{Pr}\left(G \mid R_{O}=1, \alpha_{H}\right)=0
\end{aligned}
$$

where

$$
\begin{aligned}
& \phi_{\alpha_{H}}=\operatorname{Pr}\left(G \mid \alpha_{H}\right)=\frac{\gamma \phi}{\gamma \phi+\left[\gamma+(1-\gamma) \tau_{X}\right](1-\phi)}<\phi \\
& \phi_{\alpha_{L}}=\operatorname{Pr}\left(G \mid \alpha_{L}\right)=\frac{(1-\gamma) \phi}{(1-\gamma) \phi+(1-\gamma)\left(1-\tau_{X}\right)(1-\phi)}>\phi
\end{aligned}
$$

\subsection{Equilibrium}

The equilibrium concept we use is Subgame Perfection. We focus on the equilibrium in a given calendar period $t$, where a government with reputation $\phi$ coexists with two generations of economic agents and potential "political troublemakers" (rioters). Output of the final good by generation $O$ is affected by the externality $\beta$ and the government's policy response $\alpha$. Generation $Y$ is a group of current consumers and future producers (i.e. at time $t+1$ ). It's mostly in this latter capacity that they care about the government's type. For expositional simplicity we get rid of the reference to the calendar period $t$, whenever possible.

Given our sequential timing we can solve for the equilibrium by backward induction. Let the lifetime payoffs to the two cohorts be denoted by $\pi$. The $Y$-cohort observes the policy $\alpha$ and whether or not the $O$ s have rioted $\left(R_{O}=0\right.$ or $\left.R_{O}=1\right)$. Since the $Y \mathrm{~s}$ are unproductive they only make political choices. The corresponding payoffs are (in

\footnotetext{
${ }^{11}$ If a type- $B$ government chose $\alpha \notin\left\{\alpha_{L}, \alpha_{H}\right\}$ it would unambiguously reveal its type to both cohorts. More on that when we describe the government's problem formally in section 2.3.
} 
expectation):

$$
\begin{array}{rcc}
\mathbf{I}_{\left(R_{O}=1\right)} E_{Y}\left(\pi \mid \phi_{0}\right)+\mathbf{I}_{\left(R_{O}=0\right)} E_{Y}\left(\pi \mid \phi^{\prime}\left(R_{O}, \alpha\right), \alpha\right)-C_{Y} & \text { if } & \tau_{Y}=1 \\
\mathbf{I}_{\left(R_{O}=1\right)} E_{Y}\left(\pi \mid \phi^{\prime}\left(R_{O}, \alpha\right), \alpha\right)+\mathbf{I}_{\left(R_{O}=0\right)} E_{Y}\left(\pi \mid \phi^{\prime}\left(R_{O}, \alpha\right), \alpha\right) & \text { if } & \tau_{Y}=0
\end{array}
$$

where $\mathbf{I}_{(\cdot)}$ is an indicator function and

$$
\begin{aligned}
E_{Y}\left(\pi \mid \phi_{0}\right)= & \phi_{0} T_{Y \mid \alpha_{L}}+\left(1-\phi_{0}\right)\left[\tau_{X}^{P I} T_{Y \mid \alpha_{H}}+\left(1-\tau_{X}^{P I}\right) T_{Y \mid \alpha_{L}}\right] \\
& +T_{O \mid \alpha_{L}}\left[\phi_{0} \gamma+\left(1-\phi_{0}\right) \gamma\left(1-\tau_{X}\right)\right] \\
& +T_{O \mid \alpha_{H}}\left[\phi_{0}(1-\gamma)+\left(1-\phi_{0}\right)\left(1-\gamma+\gamma \tau_{X}\right)\right] \\
E_{Y}(\pi \mid \phi, \alpha)= & T_{Y \mid \alpha} \\
& +T_{O \mid \alpha_{L}}\left[\phi \gamma+(1-\phi) \gamma\left(1-\tau_{X}\right)\right] \\
& +T_{O \mid \alpha_{H}}\left[\phi(1-\gamma)+(1-\phi)\left(1-\gamma+\gamma \tau_{X}\right]\right.
\end{aligned}
$$

$\tau_{X}^{P I}$ is the government's strategy when the externality $\beta$ is public information, that is, after the incumbent was kicked out of office earlier in the period. That, it turns out, only occurs when $\beta=\beta_{L}$.

If $O$ does not riot $\left(R_{O}=0\right)$, it is clearly better for $Y$ not to riot either. It cannot get rid of the government but still incurs the cost $C_{Y}$. Contrarily, if $O$ does riot $\left(R_{O}=1\right), Y$ updates the government's reputation to $\phi^{\prime}\left(R_{O}=1, \alpha\right)=0$. Hence the strategies for $Y$ are, for all $\alpha$ :

$$
\begin{aligned}
& \tau_{Y}\left(R_{O}=0, \alpha\right)=0 \\
& \tau_{Y}\left(R_{O}=1, \alpha\right)=\left\{\begin{array}{lll}
1 & \text { if } & C_{Y}<\overline{C_{Y}} \equiv E_{Y}\left(\pi \mid \phi_{0}\right)-E_{Y}(\pi \mid 0) \\
0 & \text { if } & C_{Y} \geq \overline{C_{Y}}
\end{array}\right.
\end{aligned}
$$

Given all the agents' strategies, the expectation operator $E_{Y}$ is over realizations of $\beta$. Before $Y$ moves, $O$ observes the realization of the externality $\beta$ and the government's policy $\alpha$. The expected payoffs are:

$$
\begin{aligned}
& F\left(\bar{C}_{Y}\right) E_{O}\left(\pi \mid \phi_{0}\right)+\left(1-F\left(\bar{C}_{Y}\right)\right) T_{O \mid \alpha}-C_{O} \quad \text { if } \quad \tau_{O}=1 \\
& T_{O \mid \alpha} \quad \text { if } \quad \tau_{O}=0
\end{aligned}
$$


where

$$
E_{O}\left(\pi \mid \phi_{0}\right)=\phi_{0} T_{O \mid \alpha_{L}}+\left(1-\phi_{0}\right)\left[\left(1-\tau_{X}^{P I}\right) T_{O \mid \alpha_{L}}+\tau_{X}^{P I} T_{O \mid \alpha_{H}}\right]
$$

Hence, for all $\alpha$ and $\beta$ :

$$
\tau_{O}(\beta, \alpha)=\left\{\begin{array}{lll}
1 & \text { if } & C_{O}<\bar{C}_{O}(\beta, \alpha) \equiv F\left(\bar{C}_{Y}\right)\left[E_{O}\left(\pi \mid \phi_{0}\right)-T_{O \mid \alpha}\right] \\
0 & \text { if } \quad C_{O} \geq \bar{C}_{O}(\beta, \alpha)
\end{array}\right.
$$

Recall that $\overline{C_{O}}\left(\beta_{H}, \alpha_{H}\right) \leq 0, \overline{C_{O}}\left(\beta_{H}, \alpha_{L}\right) \leq 0$ and $\overline{C_{O}}\left(\beta_{L}, \alpha_{L}\right) \leq 0$. In these three cases $\tau_{O}(\beta, \alpha)=0$ since we assume rioting costs are positive. The only case in which $O$ may find it optimal to riot is when the shock is $\beta_{L}$ and the policy is $\alpha_{H}$.

Finally, we need to analyze the governments' problems. A good government $(G)$ is concerned exclusively with social welfare. Formally, it solves the following maximization problem:

$$
\begin{aligned}
\max _{\tau_{X}(\beta), T_{O}, T_{Y}} \quad \mathbf{I}_{\left(\beta=\beta_{L}\right)} & \left\{\tau_{X}(\beta)\left[\frac{1}{2} U\left(T_{O \mid \alpha_{H}}\right)+\frac{1}{2} U\left(T_{Y \mid \alpha_{H}}\right)\right]\right. \\
& \left.+\left(1-\tau_{X}(\beta)\right)\left[\frac{1}{2} U\left(T_{O \mid \alpha_{L}}\right)+\frac{1}{2} U\left(T_{Y \mid \alpha_{L}}\right)\right]\right\} \\
+\mathbf{I}_{\left(\beta=\beta_{H}\right)} & \left\{\tau_{X}(\beta)\left[\frac{1}{2} U\left(T_{O \mid \alpha_{L}}\right)+\frac{1}{2} U\left(T_{Y \mid \alpha_{L}}\right)\right]\right. \\
& \left.+\left(1-\tau_{X}(\beta)\right)\left[\frac{1}{2} U\left(T_{O \mid \alpha_{H}}\right)+\frac{1}{2} U\left(T_{Y \mid \alpha_{H}}\right)\right]\right\} \\
\text { s.t. } \quad & X_{\beta, \alpha} \geq T_{O \mid \alpha}+T_{Y \mid \alpha} \text { for } \alpha \in\left\{\alpha_{L}, \alpha_{H}\right\}
\end{aligned}
$$

Bad governments $(B)$, on the other hand, are interested in rent extraction all the while being concerned with their reputation, i.e. the probability with which they are believed to be a $G$ type. With some (egregious) abuse of notation we can sketch $B^{\prime}$ s trade-off between contemporaneous office rents and long-term benefits from a good reputation as a dynamic program:

$$
\max _{\alpha} \Pi(\phi)=\rho(\beta, \alpha)+\Pi\left(\phi^{\prime}\right)
$$


More precisely,

$$
\begin{aligned}
\max _{\tau_{X}(\beta), T_{O}, T_{Y}} \quad \mathbf{I}_{\left(\beta=\beta_{L}\right)} & \left\{\tau _ { X } ( \beta ) \left[F _ { O } \left\langle-F_{Y} P+\left(1-F_{Y}\right)\right.\right.\right. \\
& \times\left(X_{\beta, \alpha_{H}}-T_{O \mid \alpha_{H}}-T_{Y \mid \alpha_{H}}+\Pi(0)\right\rangle \\
& +\left(1-F_{O}\right)\left\langle X_{\beta, \alpha_{H}}-T_{O \mid \alpha_{H}}-T_{Y \mid \alpha_{H}}\right. \\
& \left.\left.+\Pi\left(\phi_{\alpha_{H} \mid \tau_{X}}^{\prime}\right)\right\rangle\right] \\
& \left(1-\tau_{X}(\beta)\right)\left[X_{\beta, \alpha_{L}}-T_{O \mid \alpha_{L}}-T_{Y \mid \alpha_{L}}\right. \\
& \left.\left.+\Pi\left(\phi_{\alpha_{L}}^{\prime} \mid \tau_{X}\right)\right]\right\} \\
+\mathbf{I}_{\left(\beta=\beta_{H}\right)} & \left\{\tau _ { X } ( \beta ) \left[F _ { O } \left\langle-F_{Y} P+\left(1-F_{Y}\right)\right.\right.\right. \\
& \times\left(X_{\beta, \alpha_{L}}-T_{O \mid \alpha_{L}}-T_{Y \mid \alpha_{L}}+\Pi(0)\right\rangle \\
& +\left(1-F_{O}\right)\left\langle X_{\beta, \alpha_{L}}-T_{O \mid \alpha_{L}}-T_{Y \mid \alpha_{L}}\right. \\
& \left.\left.+\Pi\left(\phi_{\alpha_{L} \mid \tau_{X}}^{\prime}\right)\right\rangle\right] \\
& \left(1-\tau_{X}(\beta)\right)\left[X_{\beta, \alpha_{H}}-T_{O \mid \alpha_{H}}-T_{Y \mid \alpha_{H}}\right. \\
& \left.\left.+\Pi\left(\phi_{\alpha_{H} \mid \tau_{X}}^{\prime}\right)\right]\right\} \\
-\mathbf{I}_{\left(T_{\mid, \alpha}^{*} \neq T_{. \mid \alpha}\right)} & \hat{P}
\end{aligned}
$$

where $F_{O} \equiv F\left(\overline{C_{O}}\right), F_{Y} \equiv F\left(\overline{C_{Y}}\right)$, and $T_{. \mid \alpha}^{*}$ denotes the allocations to $O$ and $Y$ implemented by a good government. $X_{\beta, \alpha}-T_{O \mid \alpha}-T_{Y \mid \alpha} \geq 0$ is the rent a government can extract when the externality is $\beta$ and the tax rate is $\alpha . \Pi(\phi)$ is the continuation value of remaining in office when the government enjoys a reputation $\phi \in[0,1]$. Moreover, $\phi_{\alpha_{H} \mid \tau_{X}}^{\prime} \equiv \phi^{\prime}\left(R_{O}=0, \alpha_{H} \mid \tau_{X}\left(\beta_{L}\right), \tau_{X}\left(\beta_{L}\right)\right), \phi_{\alpha_{L} \mid \tau_{X}}^{\prime} \equiv \phi^{\prime}\left(R_{O}=0, \alpha_{L} \mid \tau_{X}\left(\beta_{L}\right), \tau_{X}\left(\beta_{L}\right)\right)$, and $\phi^{\prime}\left(R_{O}=1, \alpha_{H} \mid \tau_{X}\left(\beta_{L}\right), \tau_{X}\left(\beta_{L}\right)\right)=0$.

$P$ is the penalty a government has to pay whenever it is removed from office. If the government remains in office even though it completely revealed its $B$ type by announcing a resource allocation $T_{i \mid \alpha} \neq T_{i \mid \alpha}^{*}$, where $i \in\{O, Y\}$, it is subject to a penalty $\hat{P} \cdot{ }^{12}$

When the externality is $\beta_{H}$, the $O$-cohort has no incentive to riot when the government appropriately announces a high Pigouvian tax $\alpha_{H}$. In fact, all governments "do the right thing" under these circumstances. When $\beta=\beta_{L}$, on the other hand, bad governments are tempted to tax at the rate $\alpha_{H}$ as this enables them to extract exces-

\footnotetext{
${ }^{12}$ We make this assumption for technical reasons.
} 
sive rents. However, this distortion may unleash rioting and eventually a punishment of the government.

The government distorts $\left(\tau_{X}=1\right)$ if:

$$
\begin{aligned}
\rho\left(\beta_{L}, \alpha_{L}\right)+\Pi\left(\phi_{\alpha_{L} \mid 1}^{\prime}\right)< & F_{O}\left(1-F_{Y}\right)\left(\rho\left(\beta_{L}, \alpha_{H}\right)+\Pi(0)\right)-F_{O} F_{Y} P \\
& +\left(1-F_{O}\right)\left(\rho\left(\beta_{L}, \alpha_{H}\right)+\Pi\left(\phi_{\alpha_{H} \mid 1}^{\prime}\right)\right)
\end{aligned}
$$

where $\rho(\beta, \alpha)=X_{\beta, \alpha}-T_{O \mid \alpha}-T_{Y \mid \alpha}$.

Since $\phi_{\alpha_{H} \mid 0}^{\prime}=\phi_{\alpha_{L} \mid 0}^{\prime}=\phi$, the government decides against distorting (i.e. $\tau_{X}=0$ ) if

$$
\begin{aligned}
\rho\left(\beta_{L}, \alpha_{L}\right)+\Pi(\phi)> & F_{O}\left(1-F_{Y}\right)\left(\rho\left(\beta_{L}, \alpha_{H}\right)+\Pi(0)\right)-F_{O} F_{Y} P \\
& +\left(1-F_{O}\right)\left(\rho\left(\beta_{L}, \alpha_{H}\right)+\Pi(\phi)\right)
\end{aligned}
$$

Recall $\phi_{\alpha_{L} \mid 1}^{\prime}>\phi$ (when people believe the government is distorting, then the observational lack of distortion makes people increase the government's reputation) and $\phi_{\alpha_{H} \mid 1}^{\prime}<\phi$ (when people believe the government is distorting, then observed distortions lead people to decrease the government's reputation).

Given these updates, when condition (1) is fulfilled, and the government distorts, condition (1) cannot be satisfied. Similarly, when condition (1) is fulfilled, and the government does not distort, condition (1) cannot be satisfied. These are the conditions for an equilibrium in pure strategies.

Alternatively, neither of the two conditions is satisfied. In this case the equilibrium is in random strategies $\tau_{X}^{*}$ that fulfill the following condition:

$$
\begin{aligned}
\rho\left(\beta_{L}, \alpha_{L}\right)+\Pi\left(\phi_{\alpha_{L} \mid \tau_{X}^{*}}^{\prime}\right)= & F_{O}\left(1-F_{Y}\right)\left(\rho\left(\beta_{L}, \alpha_{H}\right)+\Pi(0)\right)-F_{O} F_{Y} P \\
& +\left(1-F_{O}\right)\left(\rho\left(\beta_{L}, \alpha_{H}\right)+\Pi\left(\phi_{\alpha_{H} \mid \tau_{X}^{*}}^{\prime}\right)\right)
\end{aligned}
$$

that is, the government is indifferent between distorting or not.

We now have all the elements and enough intuition to formally define the equilibrium.

Definition 1 A subgame perfect equilibrium consists of the government distorsion probability $\tau_{X}$, riot probabilities $\tau_{O}$ and $\tau_{Y}$ for the $O$ and $Y$ cohorts, respectively, and an updated 
government reputation $\phi^{\prime}$ such that:

1. the government and the two sectors maximize their expected utility, and

2. beliefs $\phi$ are updated using Bayes' rule, whenever possible.

With this characterization we can discuss the impact of some parameters on equilibrium distortions and inefficiencies introduced by the government. For example, as $P \rightarrow \infty$, condition (1) is trivially fulfilled: governments that are afraid of punishment never adopt distortionary policies. Less extremely, since $\frac{\partial \phi_{\alpha_{H} \mid \tau_{X}}^{\prime}}{\partial \tau_{X}}<0$ and $\frac{\partial \phi_{\alpha_{L}}^{\prime} \mid \tau_{X}}{\partial \tau_{X}}>0$ an increase in $P$ would reduce the right hand side of equation (2) and require a reduction in $\tau_{X}$ (the probability of distortion) to make the government indifferent again. ${ }^{13}$

We can also analyze what the effect of $\gamma$ (the realization probability of $\beta_{H}$ ) is in equilibrium. When $\gamma$ is low, $Y$ assigns a low probability to the need for an $\alpha_{H}$ policy. Hence, whenever $\alpha_{H}$ is observed, the reputation of the government suffers more. This deters the government from distorting when $\beta=\beta_{L}$. In other words, the government is more likely to distort when the policy $\alpha_{H}$ is a common occurrence. ${ }^{14}$

Next we dissect the effect of the reputation prior on the incentives to distort. Equation (1) is more likely to be fulfilled for high $\phi$ since the left hand side contains $\Pi(\phi)$ while the right hand side features $\left(1-F_{O}\right) \Pi(\phi)$ with $F_{O} \in[0,1]$, of course. When $\phi$ increases, it raises the left hand side relatively more than the right hand side. The intuition is that high reputation governments are more afraid of being displaced and loosing the gains from that reputation. When we solve the full dynamic program, $\Pi(\phi)$ is the endogenous function of interest.

When neither of the two conditions for a pure strategy equilibrium is fulfilled, we see the effect of the prior $\phi$ on the incentives to distort in equation (2). We know from section 2.2 that $\phi_{\alpha_{H}}^{\prime} \leq \phi$ and $\phi_{\alpha_{L}}^{\prime} \geq \phi$ with equality if and only if $\tau_{X}=0$ (i.e. bad

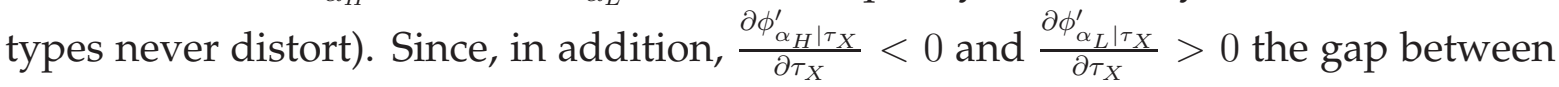

\footnotetext{
${ }^{13}$ The intuition for $\frac{\partial \phi_{\alpha_{H} \mid \tau_{X}}^{\prime}}{\partial \tau_{X}}<0$ and $\frac{\partial \phi_{L_{L} \mid \tau_{X}}^{\prime}}{\partial \tau_{X}}>0$ is as follows: the more often a government distorts, i.e. high $\tau_{X}$, the less likely $\alpha_{L}$ is a bad government's policy response. As a result, its reputation improves after $\alpha_{L}$ is observed. Conversely, as $\tau_{X}$ rises, $\alpha_{H}$ is more and more likely to be a bad - rather than a good - government's policy response with distortions.

${ }^{14}$ If the policy in fact affects the possibility of the shock in the future, for example government debt is optimal in recession, but recession is affected by indebtness, then we may have a situation of recessions and debt traps. That is, adopting $\alpha_{H}$ today increases the probability $\gamma$ of a recession tomorrow.
} 
$\phi^{\prime}\left(\alpha_{L} \mid \tau_{X}\right)$ and $\phi^{\prime}\left(\alpha_{H} \mid \tau_{X}\right)$ is increasing in $\phi$. This implies that the governments, which are most prone to distorting are those with unfavorable reputations to begin with.

\section{Effect of Redistribution on Efficiency}

Closer inspection of the equilibrium reveals that the government can discourage rioting by threatening to "bribe" sector $Y$ ex post, that is, once it observes that sector $O$ has taken to the streets. The government can in fact preempt sector- $Y$ rioting by transferring $T=\bar{C}_{Y} \equiv E_{Y}\left(\pi \mid \phi_{0}\right)-E_{Y}(\pi \mid 0)$. Given this threat, sector $O$ does not riot in the first place (since $F_{Y}=0, \bar{C}_{O}\left(\beta_{L}, \alpha_{H}\right)=0$ and $F_{O}=0$ ). This allows the government to distort with impunity, even when reputation is decreasing over time. Moreover, the mere threat of a transfer is sufficient to suppress unrest and no transfers are made on the equilibrium path. Since we grant the government an additional decision node, this result is, however, of limited relevance. More interesting is the constellation where governments decide on transfers ex ante.

\subsection{Endowment Shock}

Assume now that in addition to the stochastic externality $\beta$, the $O$-cohort receives a stochastic endowment of the final good denoted by $\theta$. The endowment realization occurs at the same time as the externality shock and can be low $\left(\theta_{L}\right)$ or high $\left(\theta_{H}\right)$ with $\operatorname{Pr}\left(\theta_{L}\right)=\delta$ and $\theta_{L}<\theta_{H}$. Like $\beta, \theta$ is observed by the old generation and the government, but not by the youngsters.

The government announces $\alpha$ and the - potentially excessive - redistribution of $\theta / 2$ at the same time. As in section 2.1.3, however, $\alpha$ and the transfers are not implemented until after both cohorts have reached their riot decision nodes in the extensive form game. As for $Y$, the cohort updates its belief about the government type in two steps:

first it updates on $\alpha$ as in section 2.2, then it updates on the observed transfer $T_{Y \mid \alpha}+\frac{\theta}{2}$. As in section 2, a good government is simply concerned with social welfare and, assuming identical welfare weights for $Y$ and $O$, will announce and implement a transfer $\theta / 2$, where $\theta \in\left\{\theta_{L}, \theta_{H}\right\}$. Bad governments, on the other hand, pursue the double objective of extracting office rents and staying in power. While $B$ types have no direct incentive to implement the socially optimal transfers, they may act responsibly 
nonetheless in an attempt to mimic a $G$ type and thereby bolster their reputation $\phi$. However, they weigh this reputational gain against the contemporaneous payoff they can realize by means of excessive redistribution.

In addition to the government's distortion strategy $\tau_{X}=\operatorname{Pr}\left(\alpha_{H} \mid \beta\right)$, we denote her redistribution strategy by:

$$
\begin{aligned}
& \tau_{R}=\operatorname{Pr}\left(\frac{\theta_{H}}{2} \mid \theta, \beta\right) \\
& \tau_{R}: \theta \times \beta \rightarrow[0,1]
\end{aligned}
$$

The timing and the strategies for $Y$ and $O$ are identical to those in section 2. $Y^{\prime}$ s and $O$ 's expected payoffs, on the other hand, now take into account the asymmetric endowment shock and we shall present the details in section 3.2.

\subsection{Equilibrium}

We can now define the equilibrium of the extended model.

Definition 2 A subgame perfect equilibrium consists of the government distorsion probability $\tau_{X}$, the probability of excessive redistribution $\tau_{R}$, riot probabilities $\tau_{O}$ and $\tau_{Y}$ for $O$ and $Y$, respectively, and an updated government reputation $\phi^{\prime}$ such that:

1. the government and the two sectors maximize their expected utility, and

2. beliefs $\phi$ are updated using Bayes' rule, whenever possible.

Now the government has an additional decision margin: redistribution. The most interesting case is a low endowment realization $\theta_{L}$, where the government has the option to implement $\frac{\theta_{H}}{2}$ in order to lower $Y^{\prime}$ s cost cutoff for rioting. $\tau_{X}$ is determined as in section 2 , that is, the government distorts $\left(\tau_{X}=1\right)$ if:

$$
\begin{aligned}
\rho\left(\beta_{L}, \alpha_{L}\right)+\Pi\left(\phi_{\alpha_{L} \mid \tau_{X}}^{\prime}\right)< & F_{O}\left(1-F_{Y}\right)\left(\rho\left(\beta_{L}, \alpha_{H}\right)+\Pi(0)\right)-F_{O} F_{Y} P \\
& +\left(1-F_{O}\right)\left(\rho\left(\beta_{L}, \alpha_{H}\right)+\Pi\left(\phi_{\alpha_{H} \mid \tau_{X}}^{\prime}\right)\right)
\end{aligned}
$$


Then, the government will decide whether to redistribute excessively $\left(\tau_{R}=1\right)$ if the payoffs from excessively redistributing the endowment (this strategy is denoted as $r$ )

$$
\left(1-F_{O}^{r}\right) \Pi\left(\phi_{r \mid \tau_{R}}^{\prime}\right)+F_{O}^{r}\left[\left(1-F_{Y}^{r}\right) \Pi(0)-F_{Y}^{r} P\right]
$$

are greater than the payoffs from NOT excessively redistributing the endowment (we denote as that strategy as $n r$ ).

$$
\left(1-F_{O}^{n r}\right) \Pi\left(\phi_{n r \mid \tau_{R}}^{\prime}\right)+F_{O}^{n r}\left[\left(1-F_{Y}^{n r}\right) \Pi(0)-F_{Y}^{n r} P\right]
$$

Hence, the government will decide whether to redistribute excessively $\left(\tau_{R}=1\right)$ if

$$
\left(1-F_{O}^{r}\right) \Pi\left(\phi_{r \mid \tau_{R}}^{\prime}\right)-\left(1-F_{O}^{n r}\right) \Pi\left(\phi_{n r \mid \tau_{R}}^{\prime}\right)>\left(F_{O}^{n r}-F_{O}^{r}\right) \Pi(0)-\left[F_{O}^{n r} F_{Y}^{n r}-F_{O}^{r} F_{Y}^{r}\right](\Pi(0)+P)
$$

We solve the problem by backward induction. For expositional simplicity, assume $\theta_{H}=\theta$ and $\theta_{L}=0$. As before, $Y^{\prime}$ 's condition for rioting is given by a cutoff $\bar{C}_{Y}=$ $E_{Y}\left(\pi \mid \phi_{0}\right)-E_{Y}(\pi \mid 0)$ where,

$$
\begin{aligned}
E_{Y}\left(\pi \mid \phi_{0}\right)= & \phi_{0} T_{Y \mid \alpha_{L}}+\left(1-\phi_{0}\right)\left[\tau_{X}^{P I} T_{Y \mid \alpha_{H}}+\left(1-\tau_{X}^{P I}\right) T_{Y \mid \alpha_{L}}\right] \\
& +T_{O \mid \alpha_{L}}\left[\phi_{0} \gamma+\left(1-\phi_{0}\right) \gamma\left(1-\tau_{X}\right)\right] \\
& +T_{O \mid \alpha_{H}}\left[\phi_{0}(1-\gamma)+\left(1-\phi_{0}\right)\left(1-\gamma+\gamma \tau_{X}\right)\right] \\
& +\frac{\theta}{2}\left[2(1-\delta)+\left(1-\phi_{0}\right) \delta \tau_{X}^{P I} \tau_{R}^{P I}-\left(1-\phi_{0}\right) \delta \gamma \tau_{X} \tau_{R}\right] \\
E_{Y}(\pi \mid \phi=0, \alpha, \theta)= & T_{Y \mid \alpha}+\frac{\theta}{2} \\
& +T_{O \mid \alpha_{L}} \gamma\left(1-\tau_{X}\right) \\
& +T_{O \mid \alpha_{H}}\left(\gamma \tau_{X}+1-\gamma\right) \\
& +\frac{\theta}{2}\left[(1-\delta)-\delta \gamma \tau_{X} \tau_{R}\right]
\end{aligned}
$$

Note that $\tau_{R}^{P I}=0$ for all reputation levels. There are no gains from redistributing in a situation where $\beta$ is known. ${ }^{15}$

Then

$$
\bar{C}_{Y}^{r}=\bar{C}_{Y}^{n r}-\frac{\theta}{2} \delta\left[1-\gamma \tau_{X}\left(\phi_{0}\right) \tau_{R}\left(\phi_{0}\right) \phi_{0}\right]
$$

\footnotetext{
${ }^{15}$ There is no effect of redistribution in terms of confusing youngsters about $\beta$ and they do not help in reducing the probability of rioting in that period, since there is no riot right after a government has been unseated.
} 
which means that with redistribution the probability youngsters enter into rioting decreases (this is $F_{Y}^{r}<F_{Y}^{n r}$ )

Similarly, the rioting decision by cohort $O$ also follows a cutoff $\bar{C}_{O} \equiv F\left(\bar{C}_{Y}\right)\left[E_{O}\left(\pi \mid \phi_{0}\right)-\right.$ $\left.\left(T_{O \mid \alpha}-\frac{\theta}{2}\right)\right]$, where

$$
E_{O}\left(\pi \mid \phi_{0}\right)=\phi_{0} T_{O, \alpha_{L}}+\left(1-\phi_{0}\right)\left[\tau_{X}^{P I} T_{O, \alpha_{H}}+\left(1-\tau_{X}^{P I}\right) T_{O, \alpha_{L}}-\frac{\theta}{2} \tau_{X}^{P I} \tau_{R}^{P I}\right]
$$

Again, since $\tau_{R}^{P I}=0$, then

$$
\bar{C}_{O}^{r}=\bar{C}_{O}^{n r}+F\left(\bar{C}_{Y}\right) \frac{\theta}{2}
$$

Whether the probability of rioting by the cohort $O$ increases or decreases depends on whether $F\left(\bar{C}_{Y}\right)$ reacts more than $\theta$ or not.

Mechanically, the way to solve for the government distorsion $\tau_{X}(\phi)$ and redistribution $\tau_{R}(\phi)$ strategies for all $\phi$ is first solving for $F\left(\bar{C}_{Y}\right)$ and $F\left(\bar{C}_{O}\right)$. As we say before, these cutoffs also depend on strategies of particular reputation levels. In the case of $\bar{C}_{Y}$, it depends on $E_{Y}\left(\pi \mid \phi_{0}\right)$ (a function of $\tau_{X}^{P I}\left(\phi_{0}\right), \tau_{R}^{P I}\left(\phi_{0}\right), \tau_{X}\left(\phi_{0}\right)$ and $\tau_{R}\left(\phi_{0}\right)$ ) and on $E_{Y}(\phi \mid 0)$ (a function of $\tau_{X}(0)$ and $\tau_{R}(0)$ ). Similarly, in the case of $\bar{C}_{O}$, it depends on $E_{O}\left(\pi \mid \phi_{0}\right)$ (a function of $\tau_{X}^{P I}\left(\phi_{0}\right)$ and $\tau_{R}^{P I}\left(\phi_{0}\right)$ ).

More specifically, the steps to characterize the government strategies (and the conditions under which they exist and are well behaved) are:

1. Solve for $\tau_{R}^{P I}\left(\phi_{0}\right), \tau_{X}^{P I}\left(\phi_{0}\right), \tau_{X}\left(\phi_{0}\right), \tau_{R}\left(\phi_{0}\right), \tau_{X}(0)$ and $\tau_{R}(0)$.

2. Determine $F\left(\bar{C}_{Y}\right)$ and $F\left(\bar{C}_{O}\right)$.

3. Solve for the taxing strategies $\tau_{X}(\phi)$ and redistribution strategies $\tau_{R}(\phi)$ for bad governments of all $\phi$.

\section{1.a. Redistribution of $\phi_{0}$ under perfect information about $\beta, \tau_{R}^{P I}\left(\phi_{0}\right)$}

As we discussed above, a just arriving bad government does not have incentives to redistribute to prevent riots in its first period (when $\beta$ is perfectly known), since there is no rioting by assumption and by redistributing would loss reputation. Then $\tau_{R}^{P I}\left(\phi_{0}\right)=0$.

\section{1.b. Distorsion of $\phi_{0}$ under perfect information about $\beta, \tau_{X}^{P I}\left(\phi_{0}\right)$}


Since a new bad government, with reputation $\phi_{0}$, does not face riots by assumption, the costs of distortion are the losses in reputation and the gains are the short term gains $\Delta \rho$

$$
\Pi\left(\phi_{\alpha_{L} \mid \tau_{X}^{P I}}^{\prime}\right)-\Pi\left(\phi_{\alpha_{H} \mid \tau_{X}^{P I}}^{\prime}\right)=\rho\left(\beta_{L}, \alpha_{H}\right)-\rho\left(\beta_{L}, \alpha_{L}\right) \equiv \Delta \rho>0
$$

The solution is $\tau_{X}^{P I} \in(0,1]$, hence governments always distort with a certain probability. New governments $B$ do not distort $\tau_{X}^{P I}=0$ if the left hand side is greater than the right hand side, which is impossible because in that case there is no updating (i.e., $\left.\alpha_{L}\left|\tau_{X}^{P I}=0=\alpha_{H}\right| \tau_{X}^{P I}=0=\phi\right)$. Contrarily, new governments $B$ do distort $\tau_{X}^{P I}=1$ if the left hand side is lower than the right hand side, which is the case if $\Pi(1)-\Pi\left(\phi_{\alpha_{H} \mid \tau_{X}^{P I}=1}^{\prime}\right)<\Delta \rho$.

\section{1.c. and 1.d: Distorsion and Redistribution of $\phi_{0}, \tau_{X}\left(\phi_{0}\right)$ and $\tau_{R}\left(\phi_{0}\right)$}

From the distortionary stage, the condition, the government will not distort $\tau_{X}\left(\phi_{0}\right)$

$$
\begin{aligned}
\Pi\left(\phi_{\alpha_{L} \mid \tau_{X}}^{\prime}\right)-\Pi\left(\phi_{\alpha_{H} \mid \tau_{X}}^{\prime}\right)> & \Delta \rho-F_{O}\left[\Pi\left(\phi_{\alpha_{H} \mid \tau_{X}}^{\prime}\right)-\Pi(0)\right] \\
& -F_{O} F_{Y}\left[\Pi(0)+P+\rho\left(\beta_{L}, \alpha_{H}\right)\right]
\end{aligned}
$$

To have a unique solution for $\tau_{X}$ for any $\phi$, we need that, the left hand side grows with $\tau_{X}$ at a higher rate than the left hand side. It is easy to see this is the case when there are no rioting possibilities. In that case the right hand side is fixed $\Delta \rho$ and the left hand side grows with $\tau_{X}$ (the higher the probability of distortion the higher the gap in reputation updating from observing a $\operatorname{tax} \alpha_{L}$ ).

In this case, the sufficient condition is $\frac{\partial L H S}{\partial \tau_{X}}>\frac{\partial R H S}{\partial \tau_{X}}$

$$
\begin{aligned}
\frac{\partial \Pi}{\partial \phi^{\prime}} \frac{\partial \phi_{\alpha_{L}}^{\prime}}{\partial \tau_{X}}-\frac{\partial \Pi}{\partial \phi^{\prime}} \frac{\partial \phi_{\alpha_{H}}^{\prime}}{\partial \tau_{X}}> & -F_{O}^{\prime} \frac{\partial \bar{C}_{O}}{\partial \tau_{X}}\left[\Pi_{\phi_{\alpha_{H}}^{\prime}}-\Pi(0)\right]-F_{O} \frac{\partial \Pi}{\partial \phi^{\prime}} \frac{\partial \phi_{\alpha_{L}}^{\prime}}{\partial \tau_{X}} \\
& -\left[F_{O}^{\prime} F_{Y} \frac{\partial \bar{C}_{O}}{\partial \tau_{X}}+F_{O} F_{Y}^{\prime} \frac{\partial \bar{C}_{Y}}{\partial \tau_{X}}\right]\left[\Pi(0)+P+\rho\left(\beta_{L}, \alpha_{H}\right)\right]
\end{aligned}
$$

where

$$
\begin{aligned}
& \frac{\partial \bar{C}_{Y}}{\partial \tau_{X}}=\phi_{0} \gamma\left(T_{\alpha_{L}}-T_{\alpha_{H}}\right)>0 \\
& \frac{\partial \bar{C}_{O}}{\partial \tau_{X}}=F_{Y}^{\prime} \frac{\partial \bar{C}_{Y}}{\partial \tau_{X}}\left[E_{O}\left(\pi \mid \phi_{0}\right)-T_{O \mid \alpha}\right]>0
\end{aligned}
$$


Then

$$
\begin{aligned}
\frac{\partial \Pi}{\partial \phi^{\prime}} \frac{\partial \phi_{\alpha_{L}}^{\prime}}{\partial \tau_{X}}-\left(1-F_{O}\right) \frac{\partial \Pi}{\partial \phi^{\prime}} \frac{\partial \phi_{\alpha_{H}}^{\prime}}{\partial \tau_{X}}> & -F_{O}^{\prime} \frac{\partial \bar{C}_{O}}{\partial \tau_{X}}\left[\Pi\left(\phi_{\alpha_{H}}^{\prime}\right)-\Pi(0)\right] \\
& -\left[F_{O}^{\prime} F_{Y} \frac{\partial \bar{C}_{O}}{\partial \tau_{X}}+F_{Y}^{\prime} F_{O} \frac{\partial \bar{C}_{Y}}{\partial \tau_{X}}\right]\left[\Pi(0)+P+\rho\left(\beta_{L}, \alpha_{H}\right)\right]
\end{aligned}
$$

Since the left hand side is positive and the right hand side is negative, this reinforces the unique equilibrium argument explored in the absence of rioting.

In the presence of redistribution, the only difference is how $\tau_{X}$ affects the probability of rioting by the young cohort. In particular, when redistribution exists,

$$
\begin{aligned}
& \frac{\partial \bar{C}_{Y}^{r}}{\partial \tau_{X}}=\phi_{0} \gamma\left[T_{\alpha_{L}}-T_{\alpha_{H}}+\frac{\theta}{2} \delta \tau_{R}\right]>\frac{\partial \bar{C}_{Y}^{n r}}{\partial \tau_{X}}>0 \\
& \frac{\partial \bar{C}_{O}^{r}}{\partial \tau_{X}}=F_{Y}^{\prime} \frac{\partial \bar{C}_{Y}}{\partial \tau_{X}}\left[E_{O}\left(\pi \mid \phi_{0}\right)-T_{O \mid \alpha}\right]>\frac{\partial \bar{C}_{O}^{n r}}{\partial \tau_{X}}>0
\end{aligned}
$$

which reinforces the uniqueness argument in the presence of redistribution. Now there are marginally more incentives to unseat the government since in the future it may not only distort taxes, but also excessively redistribute in that case.

At the redistribution stage, we assume conditions for a unique equilibrium for $\tau_{R}\left(\phi_{0}\right)$ to exist. Conditional on that result, we know there is a unique $\tau_{R}\left(\phi_{0}\right)$ that solves the equation at the redistribution stage for all other $\phi$. This is because and increase in $\tau_{R}(\phi)$ just increases the left hand side without modifying the right hand side of the following expression

$$
\left(1-F_{O}^{r}\right) \Pi\left(\phi_{r \mid \tau_{R}}^{\prime}\right)-\left(1-F_{O}^{n r}\right) \Pi\left(\phi_{n r \mid \tau_{R}}^{\prime}\right)=\left(F_{O}^{n r}-F_{O}^{r}\right) \Pi(0)-\left[F_{O}^{n r} F_{Y}^{n r}-F_{O}^{r} F_{Y}^{r}\right](\Pi(0)+P)
$$

1.e. and 1.f. Distorsion and Redistribution of $\phi=0, \tau_{X}(0)$ and $\tau_{R}(0)$

A bad government with reputation $\phi=0$ distorts $\tau_{X}(0)=1$ if

$$
F_{O} F_{Y}\left(\Pi(0)+P+\rho\left(\beta_{L}, \alpha_{H}\right)\right)<\Delta \rho
$$

and does not distorts otherwise. Note that $F_{Y}$ increases with $\tau_{X}(0)$ (since $E_{Y}(\pi \mid 0)$ decreases with $\left.\tau_{X}(0)\right)$. If the previous condition does not hold for $\tan _{X}(0)=1$, then a smaller $\tau_{X}(0)$ reduces the left hand side and makes there is a unique solution for 
$\tau_{X}(0)$

A bad government with reputation $\phi=0$ excessively redistributes $\tau_{R}(0)=1$ if

$$
F_{O}^{n r} F_{Y}^{n r}>F_{O}^{r} F_{Y}^{r}
$$

Since $F_{Y}$ increases with $\tau_{R}(0)$, we assume the conditions for a unique $\tau_{R}(0)$.

The most interesting state of nature in terms of a bad government's behavior is $\left(\beta_{L}, \theta_{L}\right)$, that is, low production externalities combined with a relatively symmetric endowment realization, which require - in the social optimum - minimal government intervention. ${ }^{16}$ By offering a resource allocation $T_{Y \mid \alpha_{H}}+\frac{\theta_{H}}{2}$ to $Y$ (which leaves $O$ with $\theta_{L}-\frac{\theta_{H}}{2}$ of the endowment), the government can extract positive rents and - at the same time - decrease the gain the youngsters may realize by way of rioting. For that reason, rioting becomes less likely and the incumbent can misbehave (that is, adopt the inefficiently high tax rate $\alpha_{H}$ ) with relative impunity. The intuition behind this result is that the government's redistribution motive is more obscure in the presence of an asymmetric stochastic endowment process. The extent to which $Y$ can disentangle the efficiency and redistribution motives depends on the precision of signal: more precise signals reduce the probability of inefficiency and excessive redistribution.

\section{Conclusion and Future Work}

Our model features a government with murky motives for redistribution, strong incentives to control resources, and a concern for its political survival (for which the reputation $\phi$ is a sufficient statistic). In addition, the citizens have asymmetric knowledge of the economic environment and political action (rioting) is costly. In this setup, governments have incentives to implement inefficient policies that boost their office rents as long as these policies do not undermine the future of their political careers. The extent to which inefficiencies occur along the equilibrium path depends on whether the symmetric information problem can be overcome: governments are more circumspect in their policy choice when they are more likely to be challenged by a large enough coalition of rioters.

When, in addition, we introduce an assymetric stochastic endowment process, the

\footnotetext{
${ }^{16}$ When $\beta_{L}=\theta_{L}=0$ the decentralized equilibrium implements the Pareto-efficient allocation.
} 
government's motives become more obscure. Transfers may be (a) designed to achieve social welfare goals or $(b)$ they are an attempt to pre-empt the removal from power. The ambiguity of transfer motives can insulate incumbents from the political consequences of an inefficient choice in the $\alpha$-dimension of the model. Precise signals about the endowment realization preclude excessive transfers altogether. On the other hand, when the signal is uninformative, governments can act inefficiently with relative impunity.

In future work, we focus on a theoretical extension of the model and on empirically testable predictions. ${ }^{17}$

In a variation of the model presented thus far, we can show that relatively unproductive sectors have a comparative advantage in "producing" votes. Clearly then, in an environment with large (small) inter-sectoral productivity differences governments have stronger (weaker) incentives to propose populist policies. For productive sectors, on the other hand, sending costly signals is comparatively less onerous and that allows them to share information about government quality more easily and frequently. Anticipating the publicization of their type, governments become more circumspect in their policy choices and soften their populist stance. In equilibrium, the incentives driven by comparative advantage balance the effects of low-cost communication. Clearly, initial conditions in terms of cross-sectoral inequality affect the trade-off, which is why not all governments are "populist". Our model can characterize this policy variation crisply.

Finally, we're planning to make progress on the empirical front by identifying salient testable predictions in the model and taking them to the times series and/or crosssectional data.

\footnotetext{
${ }^{17}$ In appendix B we present a variation of the current model featuring a continuum of sectors.
} 


\section{A Bayesian Updates}

Cohort's $Y$ update conditional on observing $R_{O}=0$ and $\alpha_{H}$ is (recall that $\phi$ denotes the prior belief about the government's quality).

$$
\begin{aligned}
& \operatorname{Pr}\left(G \mid R_{O}=0, \alpha_{H}\right)=\operatorname{Pr}\left(R_{O}=0 \mid G, \alpha_{H}\right) \operatorname{Pr}\left(G \mid \alpha_{H}\right) \\
& \left(\operatorname{Pr}\left(R_{O}=0 \mid G, \alpha_{H}\right) \operatorname{Pr}\left(G \mid \alpha_{H}\right)\right. \\
& \left.+\operatorname{Pr}\left(R_{O}=0 \mid B, \alpha_{H}\right) \operatorname{Pr}\left(B \mid \alpha_{H}\right)\right)^{-1} \\
& \operatorname{Pr}\left(R_{O}=0 \mid G, \alpha_{H}\right)=\operatorname{Pr}\left(R_{O}=0 \mid G, \alpha_{H}, \beta_{H}\right) \operatorname{Pr}\left(\beta_{H} \mid G, \alpha_{H}\right) \\
& +\operatorname{Pr}\left(R_{O}=0 \mid G, \alpha_{H}, \beta_{L}\right) \operatorname{Pr}\left(\beta_{L} \mid G, \alpha_{H}\right) \\
& =1 \times 1+\left(1-\tau_{O}\right) \times 0 \\
& =1 \\
& \operatorname{Pr}\left(R_{O}=0 \mid B, \alpha_{H}\right)=\operatorname{Pr}\left(R_{O}=0 \mid B, \alpha_{H}, \beta_{H}\right) \operatorname{Pr}\left(\beta_{H} \mid B, \alpha_{H}\right) \\
& +\operatorname{Pr}\left(R_{O}=0 \mid B, \alpha_{H}, \beta_{L}\right) \operatorname{Pr}\left(\beta_{L} \mid B, \alpha_{H}\right) \\
& =1 \times \frac{\gamma}{\tau_{X}(1-\gamma)+\gamma}+\left(1-\tau_{O}\right) \times \frac{\tau_{X}(1-\gamma)}{\tau_{X}(1-\gamma)+\gamma} \\
& =1-\frac{\tau_{O} \tau_{X}}{\tau_{X}(1-\gamma)+\gamma} \\
& \operatorname{Pr}\left(G \mid \alpha_{H}\right)=\frac{\operatorname{Pr}\left(\alpha_{H} \mid G\right) \operatorname{Pr}(G)}{\operatorname{Pr}\left(\alpha_{H} \mid G\right) \operatorname{Pr}(G)+\operatorname{Pr}\left(\alpha_{H} \mid B\right) \operatorname{Pr}(B)} \\
& =\frac{\gamma \phi}{\gamma \phi+\left[\gamma+(1-\gamma) \tau_{X}\right](1-\phi)} \\
& =\phi_{\alpha_{H}}<\phi \\
& \operatorname{Pr}\left(B \mid \alpha_{H}\right)=1-\phi_{\alpha_{H}}
\end{aligned}
$$

Putting all the pieces back together, we have:

$$
\operatorname{Pr}\left(G \mid R_{O}=0, \alpha_{H}\right)=\frac{\phi_{\alpha_{H}}}{\phi_{\alpha_{H}}+\left[1-\frac{(1-\gamma) \tau_{O} \tau_{X}}{(1-\gamma) \tau_{X}+\gamma}\right]\left(1-\phi_{\alpha_{H}}\right)}
$$


Cohort's $Y$ update conditional on observing $R_{O}=0$ and $\alpha_{L}$ is.

$$
\begin{aligned}
\operatorname{Pr}\left(G \mid R_{O}=0, \alpha_{L}\right) & =\frac{\operatorname{Pr}\left(R_{O}=0 \mid G, \alpha_{L}\right) \operatorname{Pr}\left(G \mid \alpha_{L}\right)}{\operatorname{Pr}\left(R_{O}=0 \mid G, \alpha_{L}\right) \operatorname{Pr}\left(G \mid \alpha_{L}\right)+\operatorname{Pr}\left(R_{O}=0 \mid B, \alpha_{L}\right) \operatorname{Pr}\left(B \mid \alpha_{L}\right)} \\
\operatorname{Pr}\left(R_{O}=0 \mid G, \alpha_{L}\right) & =1 \\
\operatorname{Pr}\left(R_{O}=0 \mid B, \alpha_{L}\right) & =1 \\
\operatorname{Pr}\left(G \mid \alpha_{L}\right) & =\frac{\operatorname{Pr}\left(\alpha_{L} \mid G\right) \operatorname{Pr}(G)}{\operatorname{Pr}\left(\alpha_{L} \mid G\right) \operatorname{Pr}(G)+\operatorname{Pr}\left(\alpha_{L} \mid B\right) \operatorname{Pr}(B)} \\
& =\frac{(1-\gamma) \phi}{(1-\gamma) \phi+(1-\gamma)\left(1-\tau_{G}\right)(1-\phi)} \\
& =\phi_{\alpha_{L}}>\phi \\
\operatorname{Pr}\left(B \mid \alpha_{L}\right) & =1-\phi_{\alpha_{L}}
\end{aligned}
$$

Putting all the pieces back together, we obtain:

$$
\operatorname{Pr}\left(G \mid R_{O}=0, \alpha_{L}\right)=\frac{\phi_{\alpha_{L}}}{\phi_{\alpha_{L}}+\left(1-\phi_{\alpha_{L}}\right)}=\phi_{\alpha_{L}}
$$

Given that $O$ has access to more information than $Y, R_{O}=1$ sends an unambiguous signal to the young that the government is bad. Clearly then,

$$
\begin{aligned}
& \operatorname{Pr}\left(G \mid R_{O}=1, \alpha_{L}\right)=0 \\
& \operatorname{Pr}\left(G \mid R_{O}=1, \alpha_{H}\right)=0
\end{aligned}
$$




\section{B Kitty Genovese Extension}

Instead of assuming $I=2$ as in section 2.1, assume $I \in \mathbb{Z}^{++}$. Assume also that $1 \leq I_{1}<I$ sectors are subject to a realization of $\beta$ and policy $\alpha ; I_{2}=I-I_{1}$ is the size of the new-born cohort and each of its sector's outputs is normalized to zero. For the time being, let all the sectors subject to $\beta$ and $\alpha$ be identical in terms of their action costs and productivities $\left(C^{i}=C^{j}=C_{O}\right.$ for $\left.i, j \in\left[1, I_{1}\right]\right)$. Lastly, let $C^{i}=C^{j}=C_{Y}$ for $i, j \in\left(I_{1}, I\right]$ be the symmetric action cost of all sectors in the new-born cohort.

Let $I_{\beta_{L}}^{1}\left(I_{\beta_{H}}^{1}\right)$ denote the number of sectors subject to $\beta_{L}\left(\beta_{H}\right)$. As in the baseline model, the (endogenous) policy for all sectors subject to $\beta_{H}$ is $\alpha_{H}$. Among those subject to $\beta_{L}$, the government may pick $\alpha_{L}$ for some and $\alpha_{H}$ for others: $I_{\beta_{L}, \alpha_{L}}^{1}+I_{\beta_{L}, \alpha_{H}}^{1}=I_{\beta_{L}}^{1}$.

We are interested in the behavior of the $I_{\beta_{L}, \alpha_{H}}^{1}$ sectors subject to $\beta_{L}$ and $\alpha_{H}$. Since they are homogeneous with regard to $\psi$, the expected gains from getting rid of a bad government are identical, ignoring the cost $C_{O}$. In the game, a single signal is sufficient to inform the rest of the economy about the quality of government and thereby trigger the formation of a coalition with the aim to remove the incumbent. Sending a signal, however, is costly and this is where the Kitty Genovese problem arises.

For simplicity, assume that $I_{2}+1$ participating sectors are sufficient to unseat the incumbent government. Let $\hat{\pi}_{i}$ denote sector $i$ 's gross expected gain from getting rid of the incumbent:

$$
\hat{\pi}=F\left(\overline{C_{Y}}\right)\left[E_{O}\left(\pi \mid \phi_{0}\right)-\pi(\beta, \alpha)\right]
$$

where $F\left(\bar{C}_{Y}\right)$ is defined analogously to equations (1) and (1).

Under the identity assumptions about costs and productivities, only the case $C_{O}<\hat{\pi}$ generates interesting insights.

We are looking for a symmetric mixed strategy equilibrium, where $p$ denotes the probability for each player to send a signal. If a sector sends a signal, the expected net gain is $\hat{\pi}-C_{O}$. If it does not, it realizes a net gain of zero with probability $(1-p)^{I_{1}-1}$ (no one else sends a signal) or $\hat{\pi}$ with probability $1-(1-p)^{I_{1}-1}$ (at least one sector sends a signal). 
A particular sector is indifferent whenever:

$$
\hat{\pi}-C_{O}=\hat{\pi}\left((1-p)^{I_{1}-1}\right)
$$

The (symmetric) probability for each sector is:

$$
p=1-\left(\frac{C_{O}}{\hat{\pi}}\right)^{\frac{1}{T_{1}-1}}
$$

The probability of at least one sector sending a signal is:

$$
1-(1-p)^{I_{1}}=1-\left(\frac{C_{O}}{\hat{\pi}}\right)^{\frac{I_{1}}{I_{1}-1}}
$$

In the limit with $I \rightarrow \infty$ and $I_{1} \rightarrow \infty$ the corresponding probabilities are:

$$
\begin{aligned}
p= & 0 \\
1-(1-p)^{I_{1}=} & 1-\frac{C_{O}}{\hat{\pi}}>0, \\
& \text { since } \frac{I_{1}}{I_{1}-1} \rightarrow 1 \text { as } I_{1} \rightarrow \infty
\end{aligned}
$$

That is, while the probability of any individual sending a signal goes to zero, the probability of at least one signal is strictly positive, as long as $\hat{\pi}>C_{O}$. Put differently, in the model with a continuum of sectors, the uncertainty is not completely resolved ex ante and this is reflected in the government's strategy $\tau_{X}$. 


\section{References}

Acemoglu, D. (2006): "Modeling Inefficient Institutions," in Advances in Economics and Econometrics: Theory and Applications, ed. by R. Blundell, W. K. Newey, and T. Persson, Cambridge University Press, vol. 1, 341-380.

Acemoglu, D. AND J. A. Robinson (2001): “Inefficient Redistribution," American Political Science Review, 95, 649-661.

Alesina, A. AND D. RodrIK (1994): "Distributive Politics and Economic Growth," The Quarterly Journal of Economics, 109, 465-490.

AUMANN, R. J. AND R. B. MYERSON (1988): “Endogenous formation of links between players and of coalitions: An application of the Shapley value," in The Shapley value : The Shapley value: essays in honor of Lloyd S. Shapley, ed. by A. E. Roth, Cambridge University Press.

Besley, T. And S. CoATE (1997): “An Economic Model of Representative Democracy," Quarterly Journal of Economics, 112, 85-114.

(1998): "Sources of inefficiency in a representative democracy: a dynamic analysis," American Economic Review, 88, 139-156.

CARraro, C., ed. (2003): The endogenous formation of economic coalitions, Fondazione Eni Enrico Mattei.

CAselli, F. AND M. Morelli (2004): “Bad politicians," Journal of Public Economics, $88,759-782$.

COATE, S. AND S. MORRIS (1995): “On the Form of Transfers to Special Interests," The Journal of Political Economy, 103, 1210-1235.

Persson, T. And G. TABellini (1994): “Is Inequality Harmful for Growth?” The American Economic Review, 84, 600-621.

RAY, D. (2007): A game-theoretic perspective on coalition formation, The Lipsey Lectures, Oxford University Press.

RAY, D. AND R. VOHRA (1997): “Equilibrium binding agreements," Journal of Economic Theory, 73, 30-78. 
(1999): "A theory of endogenous coalition structures," Games and Economic Behavior, 26, 286-336. 